\title{
The Results of the Paper by Westhoff et al. "Prospective Evaluation of Antibiotic Management in Ureteral Stent and Nephrostomy Interventions" Should Encourage Proper Use of Antimicrobial Prophylaxis in Patients Undergoing Nephrostomy Tube Replacement
}

\author{
Luca Ongaro Michele Rizzo Carlo Trombetta Giovanni Liguori \\ Department of Urology, Cattinara Hospital, ASUGI, University of Trieste, Trieste, Italy
}

Dear Editor,

According to the principles of antimicrobial stewardship, avoiding inappropriate antibiotics administration is a strong strategy towards containing the development and diffusion of bacterial resistances. Westhoff et al. [1] prospectively evaluated the incidence of infectious complications after 145 insertions/exchanges of ureteral stents and nephrostomies in 99 patients. The Acute Cystitis Symptom Score (ACSS) questionnaire was used to evaluate urinary tract infection symptoms before procedure and 7 days after. 122 procedures were performed without antimicrobial prophylaxis or treatment, while in 23 procedures, an antibiotic treatment or prophylaxis was administrated. Asymptomatic bacteriuria was detected in 71 and 8 cases in the 2 groups, respectively. Overall, 3 procedures required antibiotic treatment for a symptomatic urinary tract infection, body temperature $>37.5^{\circ} \mathrm{C}$ was recorded after 5 interventions, and 1 patient needed hospitalization. The authors did not find any statistically significant difference in infectious complication rates and ACSS results between procedures performed with or without antimicrobial prophylaxis or treatment. According to these results, it seems that presence of asymptomatic bacteriuria did not increase the

karger@karger.com

(c) 2021 S. Karger AG, Basel

www.karger.com/uin

Karger" risk of post-procedural infectious complications in this setting. Interestingly, ACSS questionnaire results demonstrated that transurethral procedures worsened lower urinary tract symptoms compared to percutaneous interventions, with no difference in terms of infectious complication rates between the 2 groups. This finding highlights the importance of identifying the origin of symptoms before administrating an antibiotic therapy. Given the knowledge gap on this topic, the study provides a message of clinical importance for the everyday clinical practice of many urologists. Interestingly, enough of the results of Niklas Westhoff et al. [1] are in line with our previous findings. Between 2018 and 2019, we prospectively evaluated the incidence of infectious complications in 39 patients undergoing routine office nephrostomy tube replacement performed without any antimicrobial prophylaxis. 126 procedures were analyzed [2].

We recorded 17 cases (13.5\%) of infectious complications defined as fever $\geq 38^{\circ} \mathrm{C}$. Eight patients did not require medical evaluation nor antibiotic treatment as fever resolved spontaneously, 8 cases were managed with an oral antimicrobial therapy and 1 patient required hospitalization and intravenous antimicrobial therapy. In our 
series, only a Charlson comorbidity index score $\geq 3$ was significantly associated with the risk of developing infectious complications. According to these data, we underline that using antimicrobial prophylaxis before routine nephrostomy tube replacement may be a practice based on common habits not on evidences, whereas evidence sustaining that avoiding antimicrobial prophylaxis in this scenario does not expose patients to the risk of life threatening infectious complications actually exists. Nowadays, antimicrobial resistance is challenging our health care system $[3,4]$. Currently available urological guidelines mainly refer to endourologic surgery $[5,6]$, while the utility of antimicrobial prophylaxis in patients undergoing nephrostomy tubes and ureteral stents insertion or replacement remains an understudied topic. Given the number of these procedures performed in the daily routine of every urology department worldwide, further studies producing evidence regarding proper antimicro- bial use in this setting should be encouraged in order to avoid unnecessary use of these precious drugs and help preserving their power.

\section{Conflict of Interest Statement}

The authors have no conflicts of interest to declare.

\section{Funding Sources}

No funding was requested.

\section{Author Contributions}

Luca Ongaro and Michele Rizzo detected the publication and wrote the manuscript. Carlo Trombetta and Giovanni Liguori reviewed the manuscript. All authors approved the final version of the manuscript.

\section{References}

1 Westhoff N, Anokhin A, Patroi P, Neuberger M, Siegel F, Pfalzgraf D. Prospective evaluation of antibiotic management in ureteral stent and nephrostomy interventions. Urol Int. 2021 Jul;30(7):1-8.

2 Rizzo M, Ongaro L, Claps F, Cai T, Umari P, Bucci S, et al. An observational cohort study investigating the incidence rate of infectious complications after routine office nephrostomy tube replacement without using antimicrobial prophylaxis. Urology. 2021 Jan; 147(1):27-32.
3 Wolf J. Antibiotic resistance threatens the efficacy of prophylaxis. Lancet Infect Dis. 2015 Dec;15(12):1368-9.

4 Manseck AS, Otto W, Schnabel M, Denzinger S, Burger M, Spachmann PJ. Geriatric patients and symptomatic urinary tract infections: analysis of bacterial range and resistance rates at a 3rd level of care hospital in Germany. Urol Int. 2021 Jun;25(6):1-6.
5 Dasgupta R, Grabe M. Preoperative antibiotics before endourologic surgery: current recommendations. J Endourol. 2009 Oct;23(10): 1567-70.

6 Bonkat G, Bartoletti R, Bruyere F, Cai T, Geerlings SE, Köves B, et al. Guidelines on urological infections. European Association of Urology; 2021. Available from: https:// uroweb.org/guideline/urological-infections. 